\title{
Angiotensin-(1 - 7) and Human Chorionic Gonadotropin (hCG) Modulate the Nuclear Transcription Factors or Nuclear Receptors Genes in the Tumorigenic Undifferentiated Breast Cancer Cell Line SKBR3*
}

\author{
Isidoro Binda Neto ${ }^{1,2}$, Samuel Marcos Ribeiro de Noronha ${ }^{3}$, \\ Silvana Aparecida Alves Correa de Noronha" ${ }^{3 \#}$, Maria Del Carmen Garcia Molina Wolgien", \\ Alexandre Jesus Barros ${ }^{4}$, Clovis Ryiuchi Nakaie ${ }^{4}$, Suma Imura Shimuta ${ }^{4}$, Gil Facina ${ }^{1}$, \\ Ismael Dale Cotrim Guerreiro da Silva ${ }^{1}$
}

${ }^{1}$ Laboratório de Ginecologia Molecular, Universidade Federal de São Paulo, São Paulo, Brazil; ${ }^{2}$ Universidade Federal do Estado do Rio de Janeiro, Rio de Janeiro, Brazil; ${ }^{3}$ Laboratorio de Cirurgia Translacional, Departamento de Cirurgia, UNIFESP/EPM, São Paulo, Brazil; ${ }^{4}$ Departamento de Biofísica, Universidade Federal de São Paulo, São Paulo, Brazil.

Email: ibneto@uol.com.br, labgineco@globo.com, wericabernardo@gmail.com, ${ }^{\#}$ silaac@globo.com, cnakaie@unifesp.br, sshimuta@unifesp.br, mastologista@hotmail.com, ismael.dale@gmail.com

Received May $19^{\text {th }}, 2013$; revised June $20^{\text {th }}, 2013$; accepted June $29^{\text {th }}, 2013$

Copyright (C) 2013 Isidoro Binda Neto et al. This is an open access article distributed under the Creative Commons Attribution License, which permits unrestricted use, distribution, and reproduction in any medium, provided the original work is properly cited.

\begin{abstract}
Breast cancer is the most common cancer among women. Angiotensin-(1 - 7) [Ang-(1 - 7)] has been correlated with cancer antiproliferative and apoptotic effects, similar properties of the human Chorionic Gonadotrofin (hCG). The aims of this work are to evaluate the role of Ang- $(1-7)$ and of hCG in modulating the expression of Nuclear Receptors and Coregulators related genes in the tumorigenic breast cell line SK-BR3. Three experimental groups were created: control, hCG and hCG + Ang- $(1-7)$. Cells were treated for 11 days and then had their RNA extracted. Samples were loaded into PCR Array plates containing 84 genes relate to Nuclear Receptors and Coregulators pathways. Gene expression data were used to construct canonical pathways $\left(\right.$ Metacore $\left.^{\mathrm{TM}}\right)$. hCG and hCG + Ang- $(1-7)$ treatments markedly modulate the expression of Nuclear Receptors and Coregulators related genes. hCG differentially expressed $17 \%$ of the genes, being $29 \%$ upregulated and $71 \%$ downregulated. Meanwhile, hCG + Ang-( $1-7)$ changed the expression of $30 \%$ of the genes on the plate, among these genes $56 \%$ were upregulated and $44 \%$ downregulated. Among these differentially expressed genes, we highlight Esr1, Nr2f2, and Nr2f1, Esr1, Hdac5, and Nr4A1 ( $>4$ fold). Finally MetaCore analysis based on Gene Ontology (GO) generated six networks for hCG and ten networks for the combined treatment. All generated networks are related to regulation of apoptosis or to Programmed Cell Death processes. In summary, our results herein demonstrate that the modulation of sexual hormones and of other nuclear factor genes expression might underlie the tumorigenic protection effect and the induction of cell differentiation caused by the hormones hCG and Ang-( 1 - 7), especially in Cancer Stem Cells.
\end{abstract}

Keywords: Breast Stem Cancer Cells; SK-BR3; hCG; Angiotensin-(1 - 7)

\section{Introduction}

Breast cancer is the most common cancer among women, accounting for thousands of deaths annually. In 2012, the estimated number of new breast cancer cases is above two-hundred and twenty-nine thousand [1]. Among the

\footnotetext{
"The work was supported by Grant number 2011/10516-0 and 2008/ 54383-0 from the Sao Paulo Research Foundation (FAPESP)-Brazil.

"Corresponding author.
}

various mediators that act in the carcinogenic process, the components of the renin-angiotensin system (RAS) have assumed an important role [2-5]. Angiotensin II (Ang II), better known peptide obtained from the cascade of events of RAS, has vasoconstrictive, angiogenic, hyperplastic, proliferative and metastatic properties [6,7].

Moreover, it has also been demonstrated an association between genetic polymorphisms of some RAS components with breast cancer [8-10]. Many are the evi- 
dences that the RAS is related to neoplasia of the breast tissue and also that its disruption may be involved in one or more steps that lead to carcinogenesis [11].

On the other hand, angiotensin-(1 - 7) [Ang-(1 - 7)] another peptide component of the RAS, has been extensively studied lately, for its vasodilator, antiproliferative and apoptotic effects, opposite effects generated by Ang II $[12,13]$

New breast cancer approaches have identified a small population of highly tumorigenic cells with stem cell properties in the human breast and in other solid tumors. These cells have been considered the source of tumor initiation and of its maintenance. These highly proliferative cells are referred to as cancer stem cells (CSCs) [14].

Altogether, the aims of this work are to evaluate the role of Ang-( 1 - 7) and of hCG in modulating the expression of Nuclear Receptors and Coregulators related genes in the tumorigenic breast cell line SK-BR3 in order to better understand the molecular mechanisms underlying the effects triggered by these compounds in CSCs.

\section{Methods}

\subsection{Cell Culture and Treatments}

The SKBR3 cell line was grown in DMEM supplemented with $10 \%$ FBS, $2 \mathrm{mM}$ glutamine, $100 \mathrm{U} / \mathrm{ml}$ penicillin and $100 \mu \mathrm{g} / \mathrm{ml}$ streptomycin. Three experimental groups were created: control, hCG and hCG + Ang-(1 - 7). Cells were treated for 11 days.

\subsection{RNA Extraction}

Pelleted cells were homogenized in Trizol reagent (Invitrogen) according to the manufacturer's protocol. Total RNA was purified with Qiagen RNeasy Mini Kit and subjected to treatment with DNase A. The quantity and quality of extracted RNA were measured by espectrophotometer (Nanodrop Technologies Inc., Rockland, DE).

\subsection{Real Time PCR Array}

According to the manufacturer's (Qiagen) methodology, reverse transcriptase (RT) was carried out for the synthesis of cDNA. For each sample we used as a template a PCR array plate containing 84 different pairs of primers for studying genes related to Nuclear Receptors and Coregulators pathways (RT2 Profiler ${ }^{\mathrm{TM}}$ PCR Array; SABiosciences).

\subsection{Analysis of Relevant Biological Processes and Networks by MetaCore}

The MetaCore software (GeneGo, St. Joseph, MI) is a computational resource that uses logic operations for identifying altered biological processes based upon gene expression changes. Genes with altered expression were mapped to Gene Ontology (GO) using MetaCore algorithm. GO annotations were used as indicators of biological functions. GO describes gene products in terms of their associated biological processes, cellular components, and molecular functions. The GO entries are hierarchically linked, thus allowing construction of cluster genes of crossed pathways.

\subsection{Statistical Analysis}

These results were analyzed by descriptive statistics (means and standard deviation) and inferential statistics through the student's t-test, with significance level of 5\% $(\mathrm{p}<0.05)$. Real-time PCR array reactions were processed through the online software RT2 Profiler ${ }^{\mathrm{TM}}$ PCR Array Data Analysis (SABiosciences).

\section{Results}

hCG and hCG + Ang-(1 - 7) treatments markedly modulates the expression of Nuclear Receptors and Coregulators related genes (Figures 1 and 2). hCG differentially expressed $17 \%$ of the genes, being $29 \%$ upregulated and $71 \%$ downregulated. Meanwhile, hCG + Ang-(1 - 7) changed the expression of $30 \%$ of the genes on the plate, among these $56 \%$ were upregulated and $44 \%$ downregulated. In general, the combined treatment generates a more downregulated expression profile of these genes than hCG itself (Figures 1 and 2).

Among these differentially expressed genes, we highlight Esr1, Nr2f2, and Nr2f1, Esr1, Hdac5, and Nr4A1 ( $>4$ fold) (Figure 3). Finally MetaCore analysis based on Gene Ontology (GO) generated six networks for hCG treatment and ten networks for the combined treatment. All generated networks are related to regulation of apoptosis or to Programmed Cell Death processes (Figure 4).

\section{Discussion}

The search for possible new molecular targets to treat or to early detect breast cancer is of paramount importance. Nowadays many researchers are focusing on the actions elicited by the hormone hCG, which has been shown to decrease proliferation of mammary tumor cells $[15,16]$.

The results here presented clearly demonstrate that hCG alters the expression profile of many genes encoding for proteins that act as nuclear transcription factors or as nuclear receptors.

Surprisingly both treatments restored the expression of the estrogen receptor 1 (ESR1) gene in this originally estrogen receptor negative cell line. The ESR1 is a ligand-activated transcription factor composed of several domains important for hormone binding, DNA binding, and 


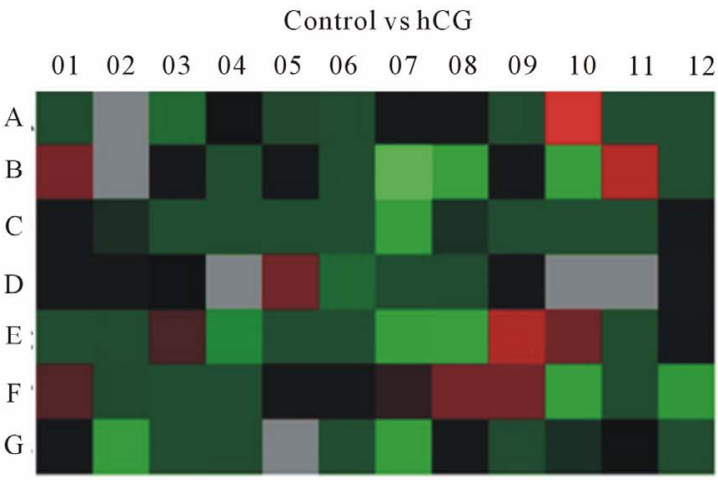

Magnitude of $\log 2$ (Fold Change)

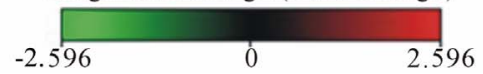

Control vs hCG + Ang-(1-7)
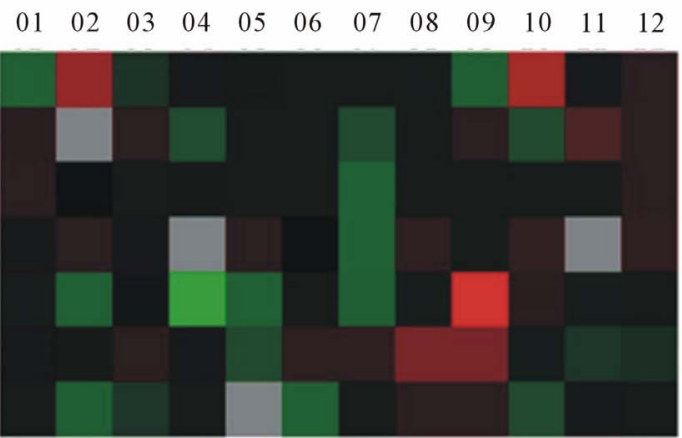

Magnitude of $\log 2$ (Fold Change)

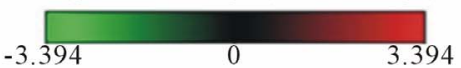

Figure 1. Heat map of SKBR3 cells gene expression after treatment.
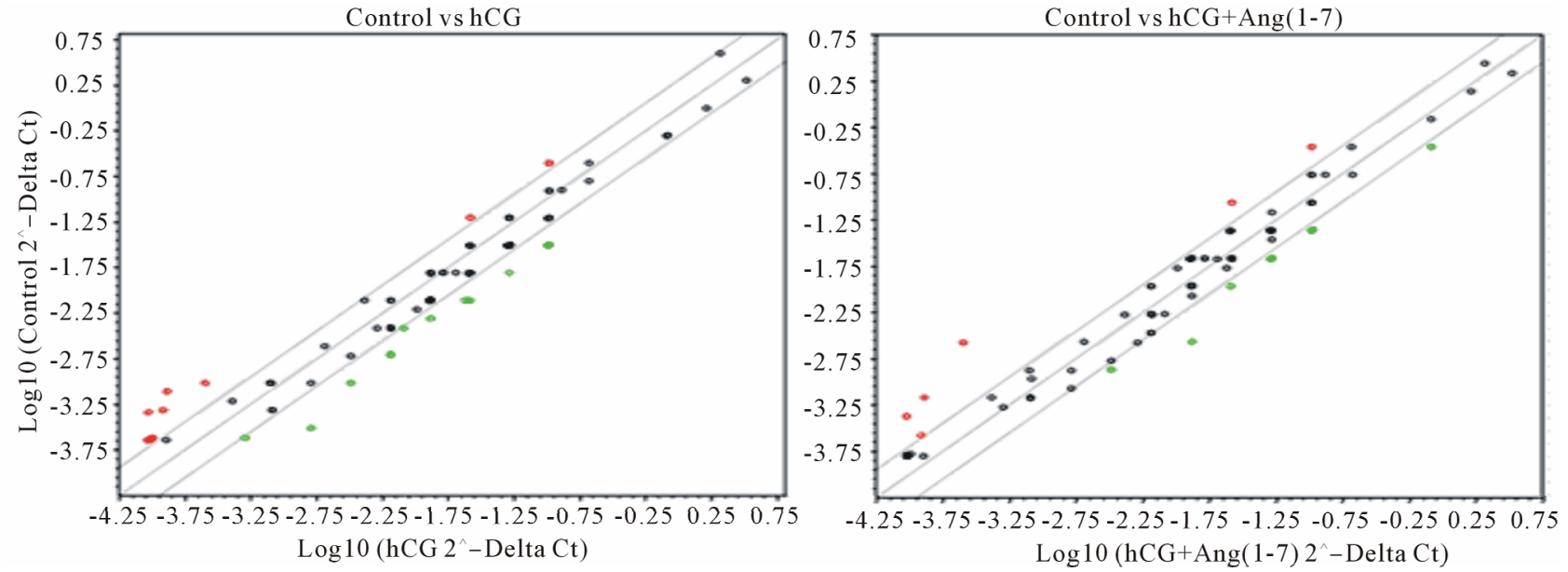

Figure 2. Gene expression analysis presented by scatter plot graphs.

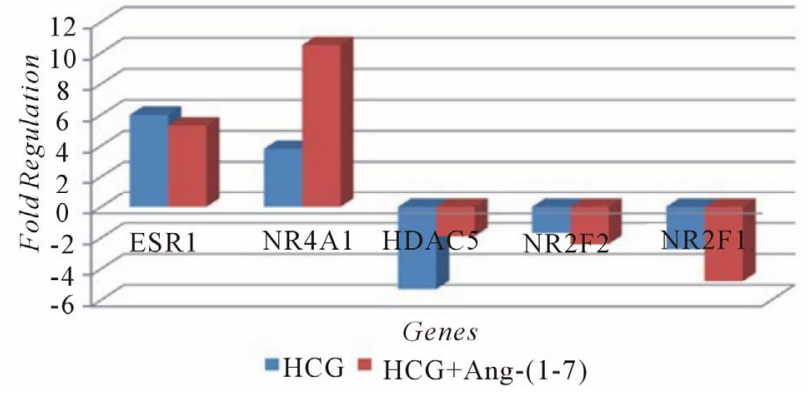

Figure 3. Most differentially expressed genes caused by both treatments in SKBR3 cells. Control cells were used as the calibrator sample.

activation of transcription. Estrogen receptors are also directly involved in different pathological processes including breast cancer, endometrial cancer, and osteoporosis [17].

Prolonged exposure to estrogens is a significant risk factor for the development of breast cancer. Estrogens exert carcinogenic effects by stimulating cell proliferation or through oxidative metabolism that forms DNA-damaging species. In SKBR3 cells, all of these estrogenforming enzymes were expressed, although the lack of ESR1 and the low levels of ESR2 expression suggest that hCG and Ang-(1 - 7) modulate the expression of sexual hormone genes [18].

Nuclear Receptor Subfamily 2 (NR2F2) encodes a member of the steroid thyroid hormone superfamily of nuclear receptors. The encoded protein is a ligand inducible transcription factor involved in regulation of many different genes (pubmed). Members of this family inhibit cell differentiation and increase cell growth. Inhibition of COUP-TFII (Nr2f2) may offer a novel therapeutic approach to breast cancer [19]. In the present study hCG downregulated COUP-TFII, which might partially explain the breast cancer protection brought about by hCG. Besides that, hCG restores ESR1 gene expression, which 


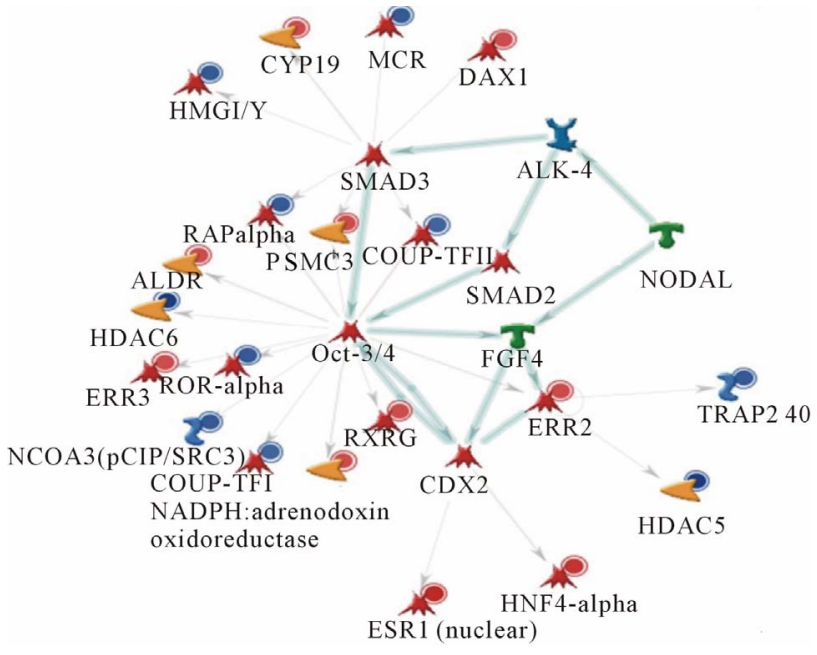

Figure 4. Top scored (by number of pathways) network generated by the active experiments. Thick cyan lines indicate the fragments of canonical pathways. Up-regulated genes are marked with red circles; down-regulated with blue circles. The checkerboard' color indicates mixed expression for the gene between files or between multiple tags for the same gene.

might be beneficial when considering the antineoplastic drugs available to treat breast cancer. At the same time, NR2F2 downregulation indicates that hCG seems to in duce cell differentiation in SKBR3 cells [19].

hCG also caused downregulation of the Nuclear Receptor Subfamily 2 (NR2F1), which may partially explain the anti-proliferative effects of this hormone [20].

Another important action of hCG was to increase the expression of the NR4A1 gene, which has an antimi-gration effect on normal cells.

Histone Deacetylase 5 (HDAC5) is an enzyme responsible for maintenance/assembly of the heterochromatin structure. As previously demonstrated its specific inhibition might contribute to increase the efficacy of DNA alteration-based cancer therapies in clinic [21]. hCG inhibits expression of HDAC5, reducing cancer progression and cell survival.

In summary, our results herein demonstrate that the modulation of sexual hormones and of other nuclear factor genes expression might underlie the tumorigenic protection effect and the induction of cell differentiation caused by the hormones hCG and Ang-(1 - 7) [22], especially in CSCs $[15,23]$.

\section{REFERENCES}

[1] American Cancer Society, "Cancer Facts \& Figures 2012," American Cancer Society, Atlanta, 2012.

[2] T. Kosaka, A. Miyajima, S. Shirotake, E. Kikuchi and M. Oya, "Phosphorylated Akt Up-Regulates Angiotensin II Type-1 Receptor Expression in Castration Resistant Pro- state Cancer," Vol. 71, No. 15, Prostate, 2011, pp. 150101517.

[3] N. Queisser, P. I. Oteiza, H. Stopper, R. G. Oli and N. Schupp, "Aldosterone Induces Oxidative Stress, Oxidative DNA Damage and NF- $\kappa$ B-Activation in Kidney Tubule Cells," Molecular Carcinogenesis, Vol. 50, No. 2, 2011, pp. 123-135. doi:10.1002/mc.20710

[4] K. Hoshino, H. Ishiguro, J. I. Teranishi, et al., "Regulation of Androgen Receptor Expression through Angiotensin II Type 1 Receptor in Prostate Cancer Cells," Prostate, Vol. 71, No. 9, 2010, pp. 964-975. doi:10.1002/pros. 21312

[5] K. Okamoto, H. Tajima and T. Ohta, et al., "The Role of Renin-Angiotensin System Independent Angiotensin II Production in Progression and Fibrosis of Intrahepatic Cholangiocarcinoma," Japanese Journal of Cancer and Chemotherapy, Vol. 37, No. 12, 2010, pp. 2231-2233.

[6] M. Fujita, I. Hayashi, S. Yamashina, M. Itoman and M. Majima, "Blockade of Angiotensin AT1a Receptor Signaling Reduces Tumor Growth, Angiogenesis, and Metastasis," Biochemical and Biophysical Research Communications, Vol. 294, No. 2, 2002, pp. 441-447. doi:10.1016/S0006-291X(02)00496-5

[7] S. Greco, A. Muscella, M. G. Elia, et al., "Angiotensin II Activates Extracellular Signal Regulated Kinases via Protein Kinase C and Epidermal Growth Factor Receptor in Breast Cancer Cells," Journal of Cellular Physiology, Vol. 196, No. 2, 2003, pp. 370-377. doi:10.1002/jep.10313

[8] S. A. Alves Correa, S. M. Ribeiro de Noronha, N. C. Nogueira-de-Souza, et al., "Association between the Angiotensin-Converting Enzyme (Insertion/Deletion) and Angiotensin II Type 1 Receptor (A1166C) Polymorphisms and Breast Cancer among Brazilian Women," Journal of the Renin-Angiotensin-Aldosterone System, Vol. 10, No. 1, 2009, pp. 51-58. doi:10.1177/1470320309102317

[9] A. P. Mendizábal-Ruiz, J. A. Morales, X. Castro Marti Nez, et al., "RAS Polymorphisms in Cancerous and Benign Breast Tissue," Journal of the Renin-AngiotensinAldosterone System, Vol. 12, No. 2, 2011, pp. 85-92. doi: $10.1177 / 1470320310383735$

[10] S. Namazi, A. Monabati, S. Ardeshir-Rouhani-Fard and N. Azarpira, "Association of Angiotensin I Converting Enzyme (Insertion/Deletion) and Angiotensin II Type 1 Receptor (A1166C) Polymorphisms with Breast Cancer Prognostic Factors in Iranian Population," Molecular Carcinogenesis, Vol. 49, No. 12, 2010, pp. 1022-1030. doi:10.1002/mc.20685

[11] M. del Pilar Carrera, M. J. Ramírez-Expósito, M. D. Mayas, M. J. García and J. M. Martínez-Martos, "Mammary Renin-Angiotensin System-Regulating Aminopeptidase Activities Are Modified in Rats with Breast Cancer," Tumor Biology, Vol. 31, No. 6, 2010, pp. 583-588. doi:10.1007/s13277-010-0072-2

[12] B. Krishnan, T. L. Smith, P. Dubey, et al., "Angiotensin(1-7) Attenuates Metastatic Prostate Cancer and Reduces Osteoclastogenesis," Prostate, Vol. 73, No. 1, 2013, pp. 71-82. doi:10.1002/pros.22542

[13] D. R. Soto-Pantoja, J. Menon, P. E. Gallagher and E.A. 
Tallant, “Angiotensin-(1-7) Inhibits Tumor Angiogenesis in Human Lung Cancer Xenografts with a Reduction in Vascular Endothelial Growth Factor," Molecular Cancer Therapeutics, Vol. 8, No. 6, 2009, pp. 1676-1683. doi:10.1158/1535-7163.MCT-09-0161

[14] P. Economopoulou, V. G. Kaklamani and K. Siziopikou, "The Role of Cancer Stem Cells in Breast Cancer Initiation and Progression: Potential Cancer Stem CellDirected Therapies," Oncologist, Vol. 17, No. 11, 2012, pp. 1394-1401. doi:10.1634/theoncologist.2012-0163

[15] S. M. R. Noronha, S. A. A. Correa-Noronha, I. H. Russo, R. L. de Cicco, J. Santucci-Pereira and J. Russo, "Human Chorionic Gonadotropin and a 15 Amino Acid hCG Fragment of the Hormone Induce Downregulation of the Cytokine IL-8 Receptor in Normal Breast Epithelial Cells," Hormone Molecular Biology and Clinical Investigation, Vol. 6, No. 3, pp. 241-245.

[16] C. A. Santos, "Papel da Angiotensina II e Angiotensina(1-7) na Proliferação E Progressão do Câncer de Mama," MsC. Thesis, Universidade Federal de São Paulo (UNIFESPEPM), São Paulo, 2013.

[17] ESR1, "Estrogen Receptor 1 [Homo sapiens (Human)]; Gene ID: 2099," 2013. http://www.ncbi.nlm.nih.gov/gene/2099

[18] N. Hevir, N. Trošt, N. Debeljak and T. L. Rižner, "Expression of Estrogen and Progesterone Receptors and Estrogen Metabolizing Enzymes in Different Breast Cancer Cell Lines," Chemico-Biological Interactions, Vol. 191, No. 1-3, 2011, pp. 206-216.

\section{doi:10.1016/j.cbi.2010.12.013}

[19] J. Qin, X. Chen, X. Xie, M. J. Tsai and S. Y. Tsai, "COUP-TFII Regulates Tumor Growth and Metastasis by Modulating Tumor Angiogenesis," Proceedings of the National Academy of Sciences of the United States of America, Vol. 107, No. 8, 2010, pp. 3687-3692. doi:10.1073/pnas.0914619107

[20] E. Moré, T. Fellner, H. Doppelmayr, et al., "Activation of the MAP Kinase Pathway Induces Chicken Ovalbumin Upstream Promoter-Transcription Factor II (COUP-TFII) Expression in Human Breast Cancer Cell Lines," Journal of Endocrinology, Vol. 176, No. 1, 2003, pp. 83-94. doi:10.1677/joe.0.1760083

[21] P. Peixoto, V. Castronovo, N. Matheus, et al., "HDAC5 Is Required for Maintenance of Pericentric Heterochromatin, and Controls Cell-Cycle Progression and Survival of Human Cancer Cells," Cell Death \& Differentiation, Vol. 19, No. 7, 2012, pp. 1239-1252. doi:10.1038/cdd.2012.3

[22] H. Kocdor, M. A. Kocdor, J. Russo, et al., "Human Chorionic Gonadotropin (hCG) Prevents the Transformed Phenotypes Induced by 17 Beta-Estradiol in Human Breast Epithelial Cells," Cell Biology International, Vol. 33, No. 11, 2009, pp. 1135-1143. doi:10.1016/j.cellbi.2009.07.002

[23] K. L. Cook, L. J. Metheny-Barlow, E. A. Tallant, et al., "Angiotensin-(1-7) Reduces Fibrosis in Orthotopic Breast Tumors," Cancer Research, Vol. 70, No. 21, 2010, pp. 8319-8328. doi:10.1158/0008-5472.CAN-10-1136 